\title{
DIFERENCIAL DE RENDIMENTOS POR NÍVEL DE ESCOLARIDADE ENTRE HOMENS E MULHERES NO BRASIL: UMA ANÁLISE DOS PRIMEIROS TRIMESTRES DE 2012, 2015 E 2019
}

\author{
Danyelle Faria de Jesus ${ }^{1}$ \\ Ana Márcia Rodrigues da Silva ${ }^{2}$ \\ Otávio Junio Faria Neves ${ }^{3}$
}

RESUMO: O nível de escolaridade das mulheres tem crescido com o passar dos anos. Paralelamente a isso, elas têm aumentado sua inserção no mercado de trabalho. Diante disso, o presente trabalho tem como objetivo analisar o diferencial de rendimentos entre homens e mulheres nos primeiros trimestres de 2012, 2015 e 2019. Para tanto, foram utilizados dados da Pesquisa Nacional por Amostra de Domicílios (PNAD) Contínua dos trimestres mencionados. A metodologia do trabalho foi elaborada por meio de estatísticas descritivas e através de uma regressão, com aplicação de uma equação minceriana. Os dados permitiram concluir que os homens ainda auferem uma renda maior que as mulheres, mesmo elas sendo mais escolarizadas, mas essa diferença salarial dos sexos tem diminuído nos trimestres analisados. Isso mostra que mesmo no período atual as mulheres sofrem discriminação no mercado de trabalho.

Palavras-Chave: Diferencial de rendimentos; Nível de escolaridade; Mercado de trabalho; Discriminação.

\section{DIFFERENTIAL INCOME BY EDUCATION LEVEL BETWEEN MEN AND WOMEN IN BRAZIL: AN ANALYSIS OF THE FIRST QUARTERS OF 2012, 2015 AND 2019}

\begin{abstract}
The education level of women has grown over the years. In parallel, they have increased their insertion in the labor market. Based on this, this article aims to analyze the income differential between men and women in the first quarters of 2012, 2015 and 2019. For this purpose, data from the Pesquisa Nacional por Amostra de Domicilios Contínua (PNAD) of the mentioned quarters were used. The methodology of the work was elaborated through descriptive statistics and through a regression, with application of a mincerian equation. The data allowed to conclude that men still earn a higher income than women, even if the female sex is more educated. However, it was found that this wage gap has narrowed in the analyzed quarters. This shows that, in today's world, women still suffer discrimination in the labor market.
\end{abstract}

Keywords: Income differential; Education level; Job market; Discrimination.

\footnotetext{
1 Graduanda em Ciências Econômicas (Unifal/MG). E-mail: danyelle_1992@hotmail.com.

2 Professora do Programa de Pós-Graduação em Economia (Unifal/MG). E-mail: anamarciarodrigues@gmail.com.

${ }_{3}$ Mestrando em Economia do Programa de Pós-Graduação em Economia (Unifal/MG). E-mail: otavionevescg@hotmail.com.
} 


\section{INTRODUÇÃO}

A participação das mulheres no mercado de trabalho sofreu diversas modificações ao longo das décadas. Segundo Ferreira et. al (2000), a inserção da mulher no mercado de trabalho ganhou força após a Segunda Guerra Mundial. Conforme Bruschini e Lombardi (1996), este movimento se intensificou após a década de 1970.

De acordo com os dados do Instituto Brasileiro de Geografia e Estatística (IBGE), o nível de escolaridade de ambos os sexos tem aumentado com o passar dos anos (IBGE, 2019). Destaca-se que as mulheres têm expandido seu nível de escolaridade e seu espaço no mercado de trabalho. Apesar disto, desde a inserção, as mulheres possuem piores condições de trabalho e menores salários.

Assim sendo, esta pesquisa procura evidenciar as diferenças salariais entre homens e mulheres por nível de escolaridade no período recente. A hipótese é que mesmo com maior nível de estudo as mulheres continuam auferindo menores rendimentos. Através disso procura-se mostrar por meio da literatura que persiste a discriminação no mercado de trabalho.

A fim de alcançar esse objetivo, serão utilizados dados da Pesquisa Nacional por Amostra de Domicílios (PNAD) Contínua dos primeiros trimestres de 2012, 2015 e 2019. A análise visa enfatizar o período mais atual de divulgação da pesquisa, isto é, 2019. É importante manter a coerência na escolha dos trimestres, visto que o mercado de trabalho sofre os efeitos da sazonalidade ao longo do ano. Por isso, foram comparados somente os primeiros trimestres. Deste modo, para comparação, foi inserido o ano de $2012 \mathrm{com}$ o intuito de incluir o primeiro trimestre de realização da pesquisa, além do primeiro trimestre de 2015. A metodologia do trabalho será composta por estatísticas descritivas e por meio de análise de regressão, com estimação de equações mincerianas. Para o melhor entendimento os dados serão demonstrados através de tabelas e gráficos.

O estudo será dividido em quatro seções, além desta introdução. Na primeira parte serão evidenciados aspectos da inserção e discriminação feminina no mercado de trabalho brasileiro, inserindo brevemente uma abordagem sobre a Teoria do Capital Humano. Na segunda seção será mostrada a metodologia aplicada para alcançar os objetivos da pesquisa e, logo em seguida, serão demonstrados os 
principais resultados do trabalho. Por último, serão apresentadas as considerações finais, ressaltando as principais conclusões do estudo.

\section{OS DIFERENCIAIS SALARIAIS E A TEORIA DO CAPITAL HUMANO}

De acordo com Arbache (2000), a lei do preço único é um dos principais postulados econômicos, segundo o qual, em situação de equilíbrio, unidades de um mesmo bem ou serviço, com as características similares precisam atingir o mesmo preço de mercado. Desta forma, no mercado de trabalho, trabalhadores com as mesmas características precisam auferir os mesmos salários. Quando esta situação não se verifica pode-se dizer que existe diferencial salarial entre os indivíduos.

Esse trabalho tem como finalidade analisar as diferenças salariais entre homens e mulheres conforme seus níveis de escolaridade. Portanto, considera-se importante o entendimento sobre a Teoria do Capital Humano. Sobre este assunto, devem-se destacar os autores precursores como Schultz (1964), Becker (1993) e Mincer (1974). Assim, será discutida brevemente esta teoria e, em seguida, apresenta-se a questão da discriminação no mercado de trabalho.

\subsection{BREVE APRESENTAÇÃO DA TEORIA DO CAPITAL HUMANO}

Essa teoria enfatiza que quanto maior o nível de escolaridade do indivíduo, maiores serão seus rendimentos ao propiciar uma maior vantagem na sua inserção no mercado de trabalho. De acordo com Schultz (1964), o investimento em educação é uma maneira de garantir um melhor posicionamento no mercado de trabalho e a expansão dos ganhos salariais conforme a escolaridade do trabalhador. Desta maneira, os diferenciais salariais são explicados em razão das diferenças educacionais dos indivíduos.

O investimento em educação é importante para os defensores dessa teoria, pois proporciona aumento de produtividade e o consequente aumento de geração de empregos com melhor qualidade, possibilitando o crescimento econômico do país. Este investimento trata-se de uma decisão individual baseada em preferências.

O aumento no capital educacional obtido pelo indivíduo, explicaria a sua posição no mercado de trabalho e os seus rendimentos (BALASSIANO; SEABRA; LEMOS, 2005). Assim sendo, de acordo com Andrade (2006), essa teoria tem como 
ideia principal salientar que o investimento em capital humano aumenta a produtividade do trabalhador, o que pode ocasionar expansão da atividade econômica e propicia maiores rendimentos auferidos pelos trabalhadores.

Segundo Biagioni (2006), essa teoria analisa os efeitos das qualificações dos trabalhadores, referentes aos seus níveis de escolaridade, na elevação de sua produtividade. Ou seja, um aumento na produtividade, elevaria o rendimento desse trabalhador, e com isso possibilitaria uma maior facilidade para entrar no mercado de trabalho.

Capital humano resulta de investimentos destinados a formação educacional e profissional dos indivíduos. Designa aptidões e habilidades pessoais que podem ser naturais ou adquiridas pela aprendizagem que possibilitam ao indivíduo auferir renda e o torna mais produtivo (ANDRADE, 2006, p.7).

Conforme os autores dessa corrente, as diferenças salariais do trabalho podem ser explicadas pelas disparidades de produtividade. A questão de o indivíduo ser mais produtivo, é explicada de acordo com suas habilidades, que proporcionam melhores salários. A teoria do capital humano apregoa que a desigualdade no âmbito do trabalho acontece devido as diferentes qualidades das pessoas (SILVA; FRANÇA; NETO, 2016). Em suma, as distintas remunerações observadas no mercado de trabalho são explicadas pelos diferenciais de produtividade entre os indivíduos.

O capital humano e a produtividade são frequentemente relacionados com os anos de estudos no sistema escolar. Entretanto, existem outras variáveis que acrescem na explicação, como: experiência profissional, reciclagem, formação profissionalizante e saúde do trabalhador (RAMOS, 1999).

É importante mencionar algumas críticas que autores realizam sobre essa teoria. A escola neoclássica enfatiza que aos aspectos ligados à oferta do trabalho determinam a produtividade, porém, os conhecimentos adquiridos na formação escolar não são determinantes na produtividade (RAMOS, 1999). As questões educacionais têm uma importante função na vida do indivíduo. Contudo, o sistema escolar não pode ser o único fator determinante para o nível da atividade econômica e para a seleção e qualificação da mão de obra. Para essa teoria os aspectos que determinam a produtividade são a inteligência, disciplina e a perseverança para vencer os obstáculos. 
Destacam-se duas críticas. A primeira delas seria que a educação é apenas uma qualificação de mão de obra para inserção no mercado de trabalho. A segunda crítica mostra que além da escolaridade, existem outros aspectos que influenciam na renda das pessoas (LIMA, 1980; ALMEIDA; PEREIRA, 2000). Dentre esses aspectos destacam os atributos pessoais de cada indivíduo.

Assim, existem outros fatores que interferem nos rendimentos além da escolaridade. Isto fica evidente, por exemplo, quando indivíduos com a mesma escolaridade e com os mesmos atributos produtivos, auferem rendimentos distintos relacionados a outros fatores como o sexo ou a raça, o que evidencia a presença de discriminação no mercado de trabalho. Na seção abaixo será evidenciada a discriminação das mulheres no mercado de trabalho brasileiro.

\title{
2.2. DISCRIMINAÇÕES POR GÊNERO NO MERCADO DE TRABALHO
}

Conforme Ferreira et. al (2001), foi durante a II Guerra Mundial que foi notado uma maior inclusão das mulheres no mercado de trabalho. Ainda de acordo com essa visão, Probst (2003) ressalta que:

\begin{abstract}
A participação da mulher no mercado de trabalho teve início com as I e II Guerras Mundiais quando os homens iam para as frentes de batalha e as mulheres passavam a assumir os negócios da família e a posição dos homens no mercado de trabalho. Mas a guerra acabou. E com ela a vida de muitos homens que lutaram pelo país. Alguns dos que sobreviveram ao conflito foram mutilados e impossibilitados de voltar ao trabalho. Foi nesse momento que as mulheres sentiram-se na obrigação de deixar a casa e os filhos para levar adiante os projetos e o trabalho que eram realizados pelos seus maridos. (PROBST, 2003, p. 2).
\end{abstract}

Antes desse período, a sociedade vivia em movimento patriarcal, onde o homem sustentava a casa e a mulher era responsável pelo serviço doméstico e cuidado com os filhos. Diante da inserção no mercado de trabalho, muitas mulheres conciliaram a realização de serviços domésticos e a maternidade, com o mercado de trabalho.

No mercado de trabalho brasileiro a inserção feminina ganhou força após os anos 1970 e não parou nos anos seguintes. Isso se deve a diversos fatores fundamentais. As mulheres ganharam espaço nas universidades, e por consequência passaram a ser mais escolarizadas. Esses fatores colaboraram para 
as mulheres atingirem novas oportunidades de trabalho (BRUSCHINI e LOMBARDI; 1996 p. 484). Segundo Silva (2014) a redução da taxa de fecundidade foi outro aspecto importante para a consolidação da mulher no mercado de trabalho.

Entre os anos de 1985 e 1995 cerca de 12 milhões de mulheres ingressaram no mercado de trabalho brasileiro, com aumento significativo de $63 \%$, conforme o estudo de Bruschini (2000). Essa transformação foi uma das mais importantes depois da década de 1970.

A partir dos anos 1990, houve um aumento significativo no nível de escolaridade da população. Diante disso, foi observado que as mulheres obtiveram e continuam tendo um nível de escolaridade superior ao dos homens. Mesmo com esse ganho de escolaridade e um maior espaço no mercado, Bruschini e Pruppin (2004) elucidam considerações importantes sobre as mulheres que ainda persiste no mercado de trabalho.

[...] ainda que essas mulheres estejam ocupando novos e promissores espaços de trabalho, nos quais sua inserção tem características bastante similares às dos homens, elas permanecem submetidas a uma desigualdade de gênero presente em todos os escalões do mercado de trabalho: ganham menos do que seus colegas de profissão (BRUSCHINI; PRUPPIN, 2004, p. 109).

De acordo com os Censos Demográficos, realizado pelo Instiuto Brasileiro de Geografia e Estatística (IBGE), o número médio de anos de estudo da população com 5 anos ou mais por sexo sofreu uma reversão apartir dos anos 1990. As mulheres passaram a ser mais escolarizadas que os homens. Antes desse período os homens tinham o nível de escolaridade superior ao das mulheres. Verificam-se os dados no Gráfico 1. 
Gráfico 1 - Número médio de anos de estudo da população com 5 anos ou mais por sexo em Censos Demográficos

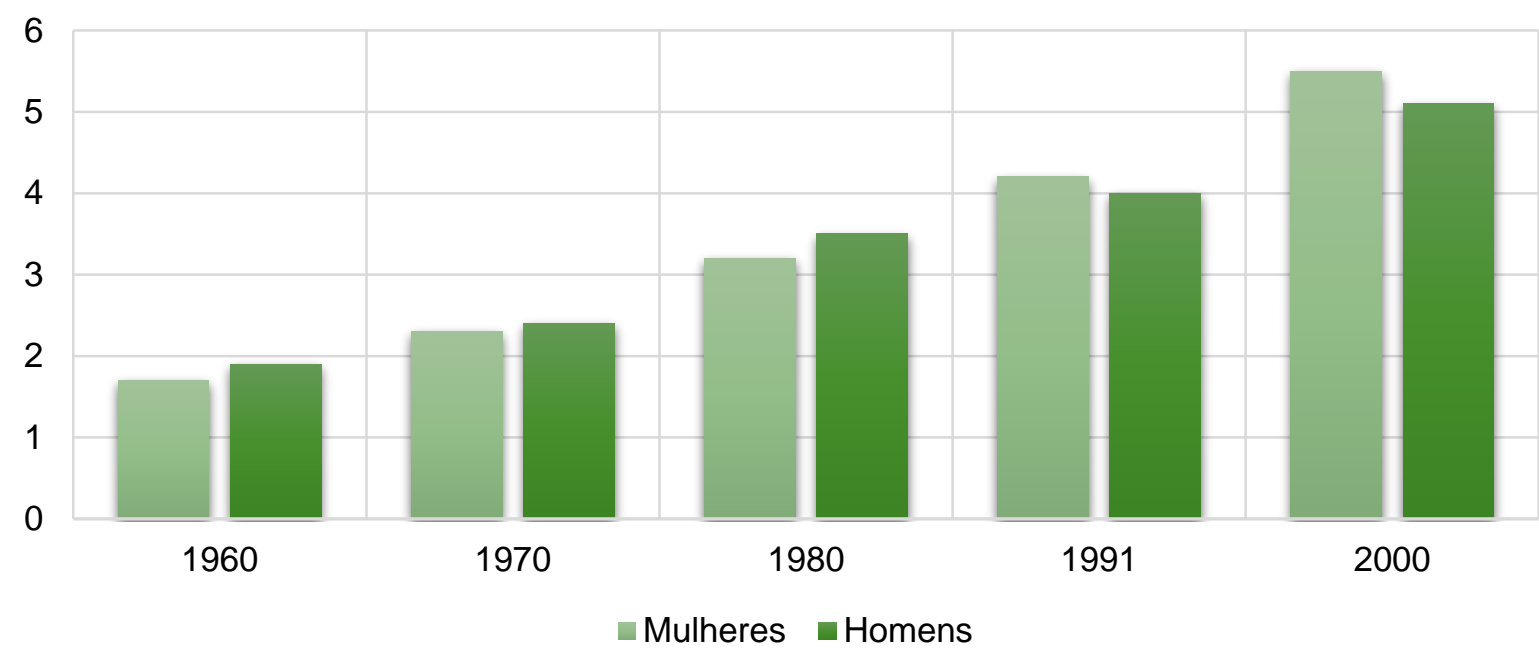

Fonte: Elaboração própria com base nos dados dos Censos Demográficos dos respectivos anos.

No Gráfico 1 nota-se que a escolaridade média das mulheres ultrapassou a dos homens a partir da década de 1990 e prosseguiu mais elevada a partir daí. Esse aumento pode ser explicado por meio da ampliação do acesso à educação ocorrida no território brasileiro nessas décadas. Além disso, essa elevação também pode ser justificada pela queda da fecundidade, das doenças e da mortalidade delas e dos filhos (BELTRÃO, 2002).

Apesar do aumento da escolaridade feminina apontado, a diferença salarial se mantém em todo território brasileiro. A maioria das mulheres continuam trabalhando com trabalhos domésticos, serviço social, educação e os homens quase não estão presentes nesses setores. O aumento da população feminina no mercado de trabalho mostrou que as trabalhadoras brasileiras encontram-se segregadas em serviços precários e com baixos rendimentos. Mesmo com o grau de escolaridade maior que dos homens, as mulheres continuam com o salário inferior e essa desigualdade se mantém no país (SILVA, 2014).

Segundo Leme e Wajnman (2000):

Não é apenas na escolaridade que homens e mulheres são diferentes. O tipo de inserção no mercado de trabalho é também bastante distinto: os homens estão mais presentes na indústria e na agricultura, enquanto as mulheres prevalecem nos serviços (LEME; WAINMAN, 2000, p.261).

Estes fatores apontam que além da discriminação, existe a segregação por gênero no mercado de trabalho, quando há funções específicas ocupadas 
majoritariamente por mulheres. Além disso, segundo Magalhães e Silva (2010), as mulheres são chamadas de "trabalhadoras de segunda categoria", isso porque há o argumento de que a dupla jornada de trabalho, com as funções domésticas e maternas, diminui o seu desempenho, o que acaba afetando a produtividade. De acordo com Bruschini (2000, p.6) "a maternidade é, sem dúvida, aquele que mais interfere no trabalho feminino, quando os filhos são pequenos."

Apesar da transformação positiva da entrada das mulheres no mercado de trabalho, as consequências têm sido marcadas por uma discriminação salarial, quando as mulheres ocupam cargos similares ao dos homens e seus rendimentos são bem inferiores. Na economia, a discriminação é entendida como um tratamento desigual em critérios desenvolvidos em uma atividade.

Em conformidade com Loureiro (2003, p. 126-127), a discriminação no mercado de trabalho vai além das diferenças salariais, caracterizando quatro tipos de discriminação, sendo elas: discriminação de trabalho, discriminação ao acesso de capital humano, discriminação de emprego e discriminação salarial. Abaixo será explicada de maneira objetiva cada uma delas.

1. Discriminação de trabalho: ocorre quando as pessoas discriminadas são impedidas de assumir determinados cargos, mesmo possuindo capacidades semelhantes das pessoas não discriminadas de realizar tais serviços.

2. Discriminação ao acesso de capital humano: acontece quando o trabalhador tem dificuldade de elevar sua produtividade, por intermédio da educação ou profissionalização no trabalho.

3. Discriminação de emprego: devido a situações com baixa oferta de empregos, determinados grupos são discriminados, sendo os mais afetados pelo desemprego.

4. Discriminação salarial: onde trabalhadores com o mesmo posto de trabalho recebem salários distintos.

A discriminação salarial é uma questão preocupante desde o século $X X$ e se mantém atual no contexto econômico, considerando os aspectos derivados do capital humano. Nesta teoria, educação e experiência explicam o acesso aos melhores empregos e salários, porque consideram a produtividade. Se na economia do trabalho essas características produtivas se mantiverem, e mesmo assim existirem as diferenças salariais, comprova-se que no mercado de trabalho há discriminação (SOUZA et. al, 2015). 
Com a busca de qualificação profissional por parte das mulheres, elas ganharam espaço no mercado de trabalho de forma significativa em termos de rendimentos. Isso tem colaborado para a diminuição do gap salarial entre homens e mulheres. Apesar da redução na diferença salarial, se comparada ao aumento do nível de escolaridade das mulheres em relação aos homens, essa diminuição foi pequena (SANTOS; RIBEIRO, 2006).

Conclui-se, então, que a diferença salarial entre os gêneros persiste mesmo com as mudanças no mercado de trabalho. Entre estas mudanças destacam-se a maior inserção das mulheres concomitantemente à busca de uma maior qualificação educacional por parte delas. Contudo, a existência de discriminação, faz com que sejam mantidas as discrepâncias salariais, o que justifica o estudo dessas diferenças no período atual.

\section{METODOLOGIA}

Neste estudo foram utilizados os dados dos primeiros trimestres de 2012, 2015 e 2019 extraídos da PNAD Contínua. Essa pesquisa é realizada pelo IBGE em todo território nacional e tem como objetivo captar informações sobre as características socioeconômicas e demográficas da população brasileira.

O motivo de escolha dos anos analisados foi feito para representar os diferentes cenários econômicos vivenciados pela economia brasileira. O primeiro trimestre de 2019 foi escolhido por ser o período mais atual no momento de realização desta pesquisa. Para comparação foram inseridos também os primeiros trimestres dos anos de 2012 e 2015, que representam anos de recessão econômica.

No ano de 1974, Mincer juntamente com Becker, afirmaram que a elevação do salário de um indivíduo ocorre com o aumento de suas habilidades pessoais, nível de escolaridade, capital humano e com experiências obtidas em seus trabalhos. Com o decorrer de seus estudos, Mincer, formulou uma equação, conhecida como equação minceriana, que tem como intuito verificar o salário em função dos anos de estudo e da experiência do trabalhador (OLIVEIRA et al. , 2015).

De acordo com Oliveira et.al (2015, p. 9) "esse modelo de determinação salarial desenvolvido por Mincer, apesar de seminal, continua sendo intensamente usado nas pesquisas que buscam entender, do ponto de vista do indivíduo, o retorno 
da educação e dos anos de experiência no trabalho." Posto isso, na primeira parte da análise foram feitas estatísticas descritivas e a segunda parte foi realizada uma regressão, conforme a equação Minceriana. O intuito é verificar quais são os retornos da educação em termos salariais, para elucidar as diferenças salariais entre homens e mulheres no mercado de trabalho. Essa equação é obtida da seguinte maneira:

$$
\ln Y=\mathrm{a}+b_{1} s_{i}+b_{2} j_{i}+v_{i}
$$

Em que InY é o logaritmo natural do salário; $s$ é a escolaridade do trabalhador expressa em anos de estudo; $j$ é a experiência do indivíduo; a e b। ( $(=1$ a 3) são os parâmetros a serem estimados; e $v$ é o termo de erro.

Neste trabalho foi elaborada a seguinte equação:

$$
\ln Y=\beta_{0}+\beta_{1} x_{1}+\beta_{2} x_{2}+\beta_{3} x_{3}+\beta_{4} x_{4}+\beta_{5} x_{5}+\beta_{6} x_{6}+\beta_{7} x_{7}(2)
$$

Para obter uma análise mais consistente sobre a diferença salarial (InY) entre ambos os sexos, além da escolaridade $\left(x_{1}\right)$, foram utilizadas mais quatro variáveis de controle: experiência $\left(x_{2}\right)$, idade $\left(x_{3}\right)$, raça $\left(x_{4}\right)$, posição de ocupação $\left(x_{5}\right)$ e região $\left(x_{6}\right)$.

O objetivo da regressão linear é indicar o comportamento dos salários conforme as variáveis: escolaridade, experiência, idade, raça, posição de ocupação e região. Diante disso, a variável salário representa a variável dependente e as outras variáveis são independentes. Para tanto, o intuito é investigar como as modificações nas variáveis independentes estão associadas com a variável dependente. Os $\beta_{k}$ são os parâmetros a serem estimados conforme as variáveis independentes. Por exemplo, como um aumento de dois anos de estudos pode influenciar a elevação ou redução do salário médio do trabalhador.

Foram estimadas três equações como esta para cada trimestre. A primeira foi realizada para a população total, considerando-se ambos os sexos, e para tanto, foi incluída a variável sexo $\left(x_{7}\right)$. Em seguida foram adicionadas outras duas análises: uma para mulheres e outra somente para homens, com base nas variáveis já apresentadas. Na próxima seção serão apresentados os resultados obtidos por meio deste estudo. 


\section{ANÁLISE DESCRITIVA DOS DADOS}

Nesta seção da pesquisa, o objetivo é mostrar a diferença salarial nos trimestres escolhidos neste estudo, com base em estatísticas descritivas. Assim, serão abordados ambos os sexos conforme os níveis de escolaridade e, em seguida, conforme a renda. Na Tabela1 serão apresentadas as estatísticas descritivas da escolaridade de ambos os sexos.

Tabela 1 - Estatísticas descritivas da escolaridade entre homens e mulheres no primeiro trimestre 2012, 2015 e 2019 - Brasil

\begin{tabular}{lcccccc}
\hline & \multicolumn{2}{c}{ 2012 } & \multicolumn{2}{c}{ 2015 } & \multicolumn{2}{c}{ 2019 } \\
\hline Escolaridade & Homens & Mulheres & Homens & Mulheres & Homens & Mulheres \\
Média & 7,54 & 8,05 & 7,93 & 8,47 & 8,43 & 8,95 \\
Desvio Padrão & 4,70 & 4,83 & 4,68 & 4,82 & 4,72 & 4,85 \\
Percentil 1 & 0 & 0 & 0 & 0 & 0 & 0 \\
Percentil 25 & 4 & 4 & 5 & 5 & 5 & 5 \\
Percentil 50 & 8 & 9 & 8 & 9 & 9 & 10 \\
Percentil 90 & 13 & 15 & 14 & 16 & 15 & 16 \\
CV $^{4}$ & 0,62 & 0,60 & 0,59 & 0,57 & 0,56 & 0,54 \\
Mínimo & 0 & 0 & 0 & 0 & 0 & 0 \\
Máximo & 16 & 16 & 16 & 16 & 16 & 16 \\
\hline
\end{tabular}

Fonte: Elaborado pelos autores conforme dados das PNADs de 2012, 2015, 2019.

A diferença no nível de escolaridade entre homens e mulheres é facilmente notada nos anos analisados. As mulheres possuem um grau de anos de estudo superior aos homens no Brasil. Podem-se notar diferenças nos $90 \%$ da população mais escolarizada. Enquanto no primeiro trimestre de 2012 os homens dessa faixa analisada possuíam até 13 anos de estudo, as mulheres tinham até 15 anos. No primeiro trimestre de 2019 , essa diferença diminuiu, mas as mulheres se mantiveram mais escolarizadas, com 16 anos de estudo contra 15 anos para os homens.

Outro fator a ser analisado é o coeficiente de variação, que teve queda ao longo dos anos, tanto para os homens quanto para as mulheres. Com esse dado pode-se dizer que a dispersão da escolaridade mesmo que aos poucos vem diminuindo. Com base nos dados da Tabela 1 observa-se ainda que o nível de escolaridade para ambos os sexos vem aumentando no país. Na Tabela 2 estão apresentadas as estatísticas descritivas da renda do trabalho conforme o sexo.

\footnotetext{
${ }^{4} \mathrm{CV}=$ coeficiente de variação
} 
Tabela 2 - Estatísticas descritivas da renda nominal do trabalho entre homens e mulheres nos primeiros trimestres 2012, 2015 e $2019^{\star}$ - Brasil

\begin{tabular}{lcccccc}
\hline & \multicolumn{2}{c}{ 2012 } & \multicolumn{2}{c}{ 2015 } & \multicolumn{2}{c}{2019} \\
\hline & Homens & Mulheres & Homens & Mulheres & Homens & Mulheres \\
\hline Renda Média & 1544,93 & 1199,36 & 1897,02 & 1514,37 & 2499,25 & 2110,06 \\
Desvio Padrão & 2420,40 & 1678,19 & 2780,19 & 2040,77 & 3975,94 & 3339,70 \\
Percentil 1 & 0 & 0 & 0 & 0 & 0 & 0 \\
Percentil 50 & 950 & 740 & 1200 & 1000 & 1500 & 1300 \\
Percentil 90 & 3000 & 2480 & 3500 & 3000 & 5000 & 4000 \\
CV & 1,57 & 1,40 & 1,46 & 1,35 & 1,59 & 1,58 \\
Mínimo & 0 & 0 & 0 & 0 & 0 & 0 \\
Máximo & 180000 & 60000 & 100000 & 60000 & 200000 & 130000 \\
\hline
\end{tabular}

Fonte: Elaborado pelos autores conforme os dados das PNADs de 2012, 2015, 2019. *Renda em $\mathrm{R} \$$.

De acordo com as estatísticas descritivas da renda nominal ${ }^{5}$ do trabalho, a renda média dos homens é superior à das mulheres em todos os trimestres analisados. O desvio padrão na renda dos homens é maior, o que indica que a disparidade nos rendimentos dos homens é maior do que entre as mulheres. Ou seja, mesmo auferindo menor renda do trabalho, as mulheres têm salários mais homogêneos. Um ponto que chama a atenção é a discrepância das rendas entre o percentil 90 e os salários máximos no país, conforme captado pelas PNADs.

No primeiro trimestre de 2019 pode-se observar que os homens chegaram a receber $R \$ 200.000,00$, enquanto $90 \%$ da população masculina recebia até $R \$$ $5.000,00$. No caso das mulheres $90 \%$ delas eram remuneradas em até $R \$ 4.000,00$ e o rendimento máximo chega a $\mathrm{R} \$ 130.000,00$. Esses dados além de mostrar uma diferença salarial entre os sexos apontam outro problema recorrente no Brasil, a grande desigualdade de renda.

Ao verificar as tabelas conjuntamente, observa-se que as mulheres têm um nível de escolaridade médio maior que os homens. Se fosse levar em consideração a teoria do capital humano, as mulheres teriam que auferir uma renda maior, pois essa teoria apregoa que quanto maior a escolaridade maior será sua renda. Mas, os dados dessa pesquisa demonstram que os homens têm um nível de escolaridade inferior e recebem uma renda maior.

\footnotetext{
${ }^{5}$ Renda do trabalho sem descontar a inflação do período.
} 
Gráfico 2 - Média do nível de escolaridade entre homens e mulheres nos primeiros trimestres de 2012, 2015 e 2019 - Brasil

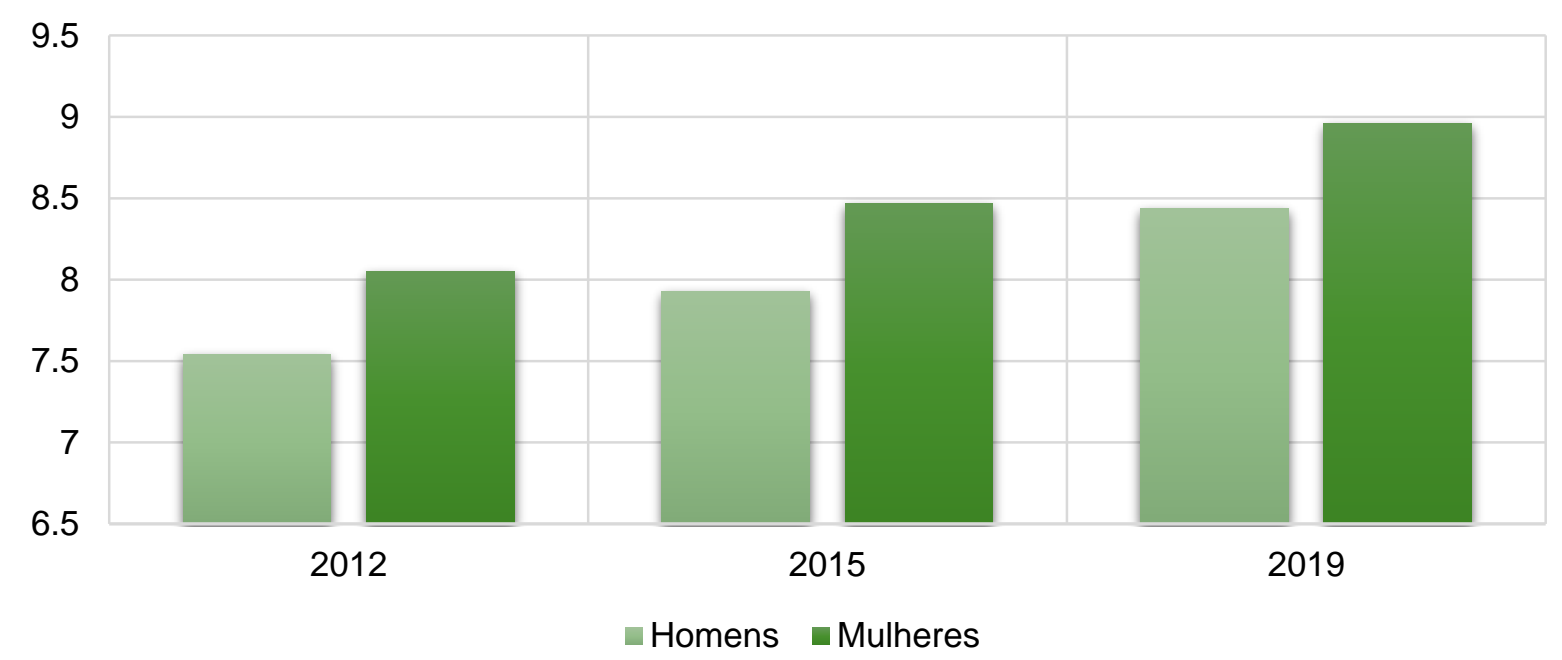

Fonte: Elaborado pelos autores conforme os dados das PNADs de 2012, 2015, 2019.

No gráfico acima pode-se observar que a escolaridade média feminina é cerca de 6,8\% maior do que a escolaridade masculina no primeiro trimestre de 2012. Essa média se manteve no primeiro trimestre de 2015. No primeiro trimestre de 2019, essa diferença caiu, mas as mulheres continuaram mais escolarizadas do que os homens, cerca de $6,2 \%$. Além disso, o nível de escolaridade de ambos os sexos tem evoluído a cada período analisado. No gráfico 3 será apresentado a renda média real para ambos os sexos.

Gráfico 3 - Média dos rendimentos reais de homens e mulheres nos primeiros trimestres de 2012, 2015 e 2019 - Brasil $(e m R \$)^{*}$

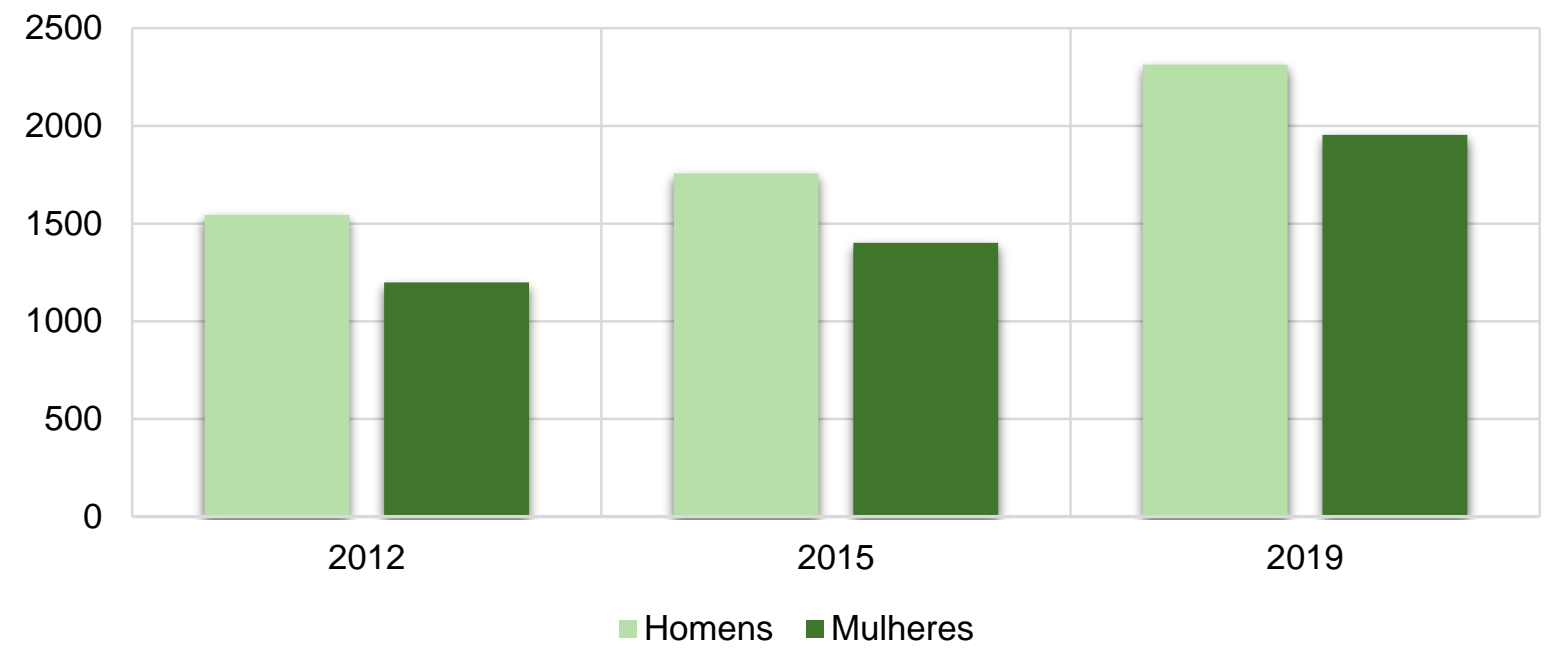

Fonte: Elaborado pelos autores conforme os dados das PNADs de 2012, 2015, 2019. *Renda real. 
Com base nas rendas médias reais de ambos os sexos, analisa-se que a diferença salarial tem caído com o passar dos trimestres analisados. No primeiro trimestre de 2012 o salário médio dos homens era cerca de 28,3\% maior que o salário médio das mulheres. Em 2015 essa diferença foi menor, cerca de 25,3\% a mais nos rendimentos masculinos. Já em 2019, os homens passaram a ganhar $18,4 \%$ a mais que as mulheres, evidenciando uma queda na diferença salarial. Demonstradas as estatísticas descritivas, a seguir serão apresentadas as equações mincerianas para o período de análise já salientado.

\section{ANÁLISES DOS DIFERENCIAIS DE RENDIMENTOS: EQUAÇÕES MINCERIANAS}

Através das estimações das equações mincerianas foram elaboradas tabelas com uma regressão geral com a inclusão da variável sexo e mais duas regressões adicionais separadamente para homens e para mulheres, conforme a equação apresentada na metodologia. Em todas as tabelas por default a primeira categoria de cada variável foi considerada como categoria de referência. Por este motivo não são apresentados coeficientes para estas variáveis.

Para a análise dos dados, será observado como as variáveis escolhidas nesta pesquisa influenciam o salário. Diante disso, na Tabela 3 serão apresentados os dados para o primeiro trimestre de 2012.

Ao observar a Tabela 3, verifica-se que após 2 anos de estudo, quanto maior o nível de escolaridade maior será o salário. Comparando homens e mulheres conclui-se que a partir do terceiro ano de estudo, a renda aumenta em todos os anos decorrentes para ambos, mas as variações são sempre maiores para os homens. Enquanto com 16 anos de estudo o salário das mulheres aumenta cerca de 156\% em relação aos analfabetos, o dos homens aumenta 165\%. Nesse caso a escolaridade tem uma interferência maior na renda dos homens cerca de 5,8\%. 
Tabela 3 - Análise da equação minceriana para o primeiro trimestre de 2012

\begin{tabular}{|c|c|c|c|}
\hline Lnsalário & População & Homens & Mulheres \\
\hline Escolaridade & \multicolumn{3}{|c|}{ Coeficientes } \\
\hline \multicolumn{4}{|l|}{ Sem instrução e menos de 1 ano de } \\
\hline 1 ano de estudo & $0,174^{*}$ & $0,190^{*}$ & $0,144^{*}$ \\
\hline 2 anos de estudo & $0,112^{*}$ & $0,149^{*}$ & $0,024^{\star *}$ \\
\hline 3 anos de estudo & $0,171^{*}$ & $0,190^{*}$ & $0,136^{*}$ \\
\hline 4 anos de estudo & $0,246^{*}$ & $0,271^{*}$ & $0,200^{*}$ \\
\hline 5 anos de estudo & $0,365^{*}$ & $0,389^{*}$ & $0,326^{*}$ \\
\hline 6 anos de estudo & $0,432^{*}$ & $0,457^{*}$ & $0,396^{*}$ \\
\hline 7 anos de estudo & $0,480^{*}$ & $0,512^{*}$ & $0,434^{*}$ \\
\hline 8 anos de estudo & $0,505^{*}$ & $0,519^{*}$ & $0,490^{*}$ \\
\hline 9 anos de estudo & $0,556^{*}$ & $0,561^{*}$ & $0,555^{*}$ \\
\hline 10 anos de estudo & $0,523^{*}$ & $0,529^{*}$ & $0,516^{*}$ \\
\hline 11 anos de estudo & $0,581^{*}$ & $0,573^{*}$ & $0,587^{*}$ \\
\hline 12 anos de estudo & $0,801^{*}$ & $0,808^{*}$ & $0,780^{*}$ \\
\hline 13 anos de estudo & $0,930^{*}$ & $0,946^{*}$ & $0,889^{*}$ \\
\hline 14 anos de estudo & $1,065^{\star}$ & $1,064^{*}$ & $1,044^{*}$ \\
\hline 15 anos de estudo & $1,145^{\star}$ & $1,166^{*}$ & $1,104^{*}$ \\
\hline 16 anos de estudo & $1,611^{*}$ & $1,651^{*}$ & $1,561^{*}$ \\
\hline \multicolumn{4}{|l|}{ Experiência } \\
\hline \multicolumn{4}{|l|}{ Menos de 1 mês ${ }^{\star * *}$} \\
\hline De 1 mês a menos de 1 ano & $0,237^{*}$ & $0,254^{*}$ & $0,213^{*}$ \\
\hline De 1 ano a menos de 2 anos & $0,325^{*}$ & $0,325^{*}$ & $0,324^{*}$ \\
\hline 2 anos ou mais & $0,448^{*}$ & $0,445^{*}$ & $0,458^{*}$ \\
\hline \multicolumn{4}{|l|}{ Idade } \\
\hline & $0,008^{*}$ & $0,010^{*}$ & $0,005^{*}$ \\
\hline \multicolumn{4}{|l|}{ Sexo } \\
\hline Homens $^{* \star *}$ & & & \\
\hline Mulheres & $-0,407^{*}$ & & \\
\hline \multicolumn{4}{|l|}{ Raça } \\
\hline \multicolumn{4}{|l|}{ Branca*** $^{*}$} \\
\hline Preta & $-0,086^{*}$ & $-0,101^{*}$ & $-0,067^{*}$ \\
\hline Amarela & $0,012^{\star *}$ & $-0,026^{\star *}$ & $0,068^{*}$ \\
\hline Parda & $-0,101^{*}$ & $-0,115^{\star}$ & $-0,085^{\star}$ \\
\hline Indígena & $-0,085^{\star}$ & $-0,067^{\star}$ & $-0,109^{*}$ \\
\hline \multicolumn{4}{|l|}{ Setor de atividade } \\
\hline \multicolumn{4}{|l|}{ Agricultura, pecuária, produção } \\
\hline Indústria geral & $0,504^{*}$ & $0,561^{*}$ & $0,490^{*}$ \\
\hline Construção & $0,468^{*}$ & $0,453^{*}$ & $0,647^{*}$ \\
\hline Comércio, reparação de veículos & $0,405^{\star}$ & $0,395^{*}$ & $0,510^{*}$ \\
\hline Transporte, armazenagem e correio & $0,549^{*}$ & $0,536^{*}$ & $0,668^{*}$ \\
\hline Alojamento e alimentação & $0,397^{*}$ & $0,315^{*}$ & $0,555^{\star}$ \\
\hline Informação, comunicação e atividades & $0,533^{*}$ & $0,477^{*}$ & $0,699^{*}$ \\
\hline Administração pública, defesa e & $0,708^{*}$ & $0,692^{*}$ & $0,828^{*}$ \\
\hline Educação, saúde humana e serviços & $0,514^{*}$ & $0,429^{*}$ & $0,651^{*}$ \\
\hline Outros Serviços & $0,257^{*}$ & $0,217^{*}$ & $0,377^{*}$ \\
\hline Serviços domésticos & $0,177^{*}$ & $0,081^{*}$ & $0,296^{*}$ \\
\hline Atividades mal definidas & $0,202^{*}$ & $0,212^{*}$ & $0,274^{*}$ \\
\hline \multicolumn{4}{|l|}{ Região } \\
\hline \multicolumn{4}{|l|}{ Norte $^{\star \star \star}$} \\
\hline Nordeste & $-0,232^{*}$ & $-0,212^{*}$ & $-0,258^{*}$ \\
\hline Sudeste & $0,190^{*}$ & $0,193^{*}$ & $0,184^{*}$ \\
\hline Sul & $0,219^{*}$ & $0,230^{*}$ & $0,204^{*}$ \\
\hline Centro-Oeste & $0,253^{*}$ & $0,293^{*}$ & $0,194^{*}$ \\
\hline Constante & $5,101^{*}$ & $5,018^{*}$ & $4,726^{*}$ \\
\hline
\end{tabular}

Fonte: Elaborado pelos autores conforme os dados da PNAD de 2012. * $p$-valor menor que 0,05 (significante). ${ }^{* *} p$-valor maior que 0,05 (não significante). ${ }^{* * *}$ Categorias de referência. 
Em relação à experiência, pode-se dizer que quanto maior o tempo de trabalho maior será a variação da renda. Com dois anos ou mais de experiência em relação a quem tem menos de um mês de trabalho, o salário dos homens sobe em média $44,5 \%$ enquanto o das mulheres tem uma variação ainda maior de aproximadamente $45,8 \%$.

A variável idade pode ser analisada da seguinte forma, a cada um ano a mais de idade varia $0,8 \%$ do salário da população total. Quando analisado homens e mulheres separadamente, percebe-se que a renda do homem varia $50 \%$ a mais a cada ano de idade se comparado as mulheres.

Com base na variável "sexo", as mulheres ganham salários cerca de $40 \%$ a menos do que os homens, apenas pelo fato de serem mulheres, já que as variáveis de controle foram inseridas no modelo apresentado. Isso mostra que a discriminação está presente no mercado de trabalho e afeta de forma expressiva a renda das mesmas.

Usando os brancos como categoria de referência da variável raça, pode-se observar que as variações nos rendimentos dos negros, pardos e indígenas são negativas, isso significa que os brancos têm rendimentos superiores. Os amarelos possuem coeficientes maiores, porém seu nível de significância não está de acordo com as estatísticas da pesquisa. Analisando separadamente, pode-se dizer que a raça afeta mais o salário dos homens do que das mulheres, isso pode ser explicado por conta de uma maior homogeneidade na renda feminina.

Quanto ao setor de atividade os trabalhos voltados para a administração pública, defesa e seguridade social são os mais rentáveis para ambos os sexos. $A$ análise é feita com base na agricultura e pecuária, que de acordo com os dados é a menos rentável para os trabalhadores.

Outra variável que interfere na variação do salário é a região onde reside o trabalhador. Com base na região Norte apenas a região Nordeste possui níveis menores de rendimento, as outras regiões possuem coeficientes positivos para a renda do indivíduo. A variação no salário do homem é maior no Centro-Oeste e das mulheres na região Sul do país. 
Tabela 4 - Análise da equação minceriana para o primeiro trimestre de 2015

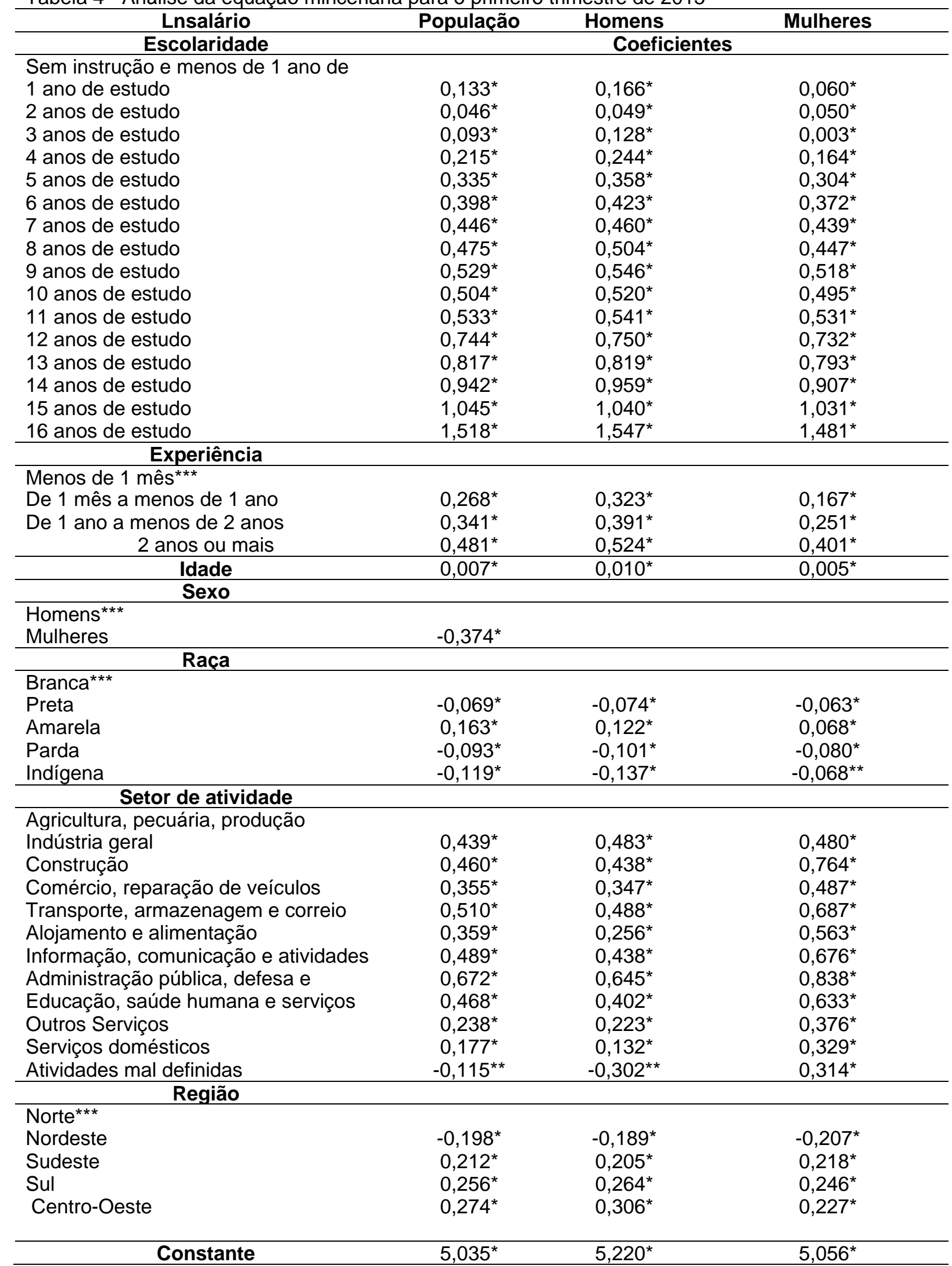

Fonte: Elaborado pelos autores conforme os dados da PNAD de 2015. ${ }^{*} \mathrm{p}$-valor menor que 0,05 (significante). ${ }^{* *} p$-valor maior que 0,05 (não significante). ${ }^{* * *}$ Categorias de referência. 
Com base no primeiro trimestre de 2015, pode-se observar que houve uma queda considerável na variação da renda por meio da escolaridade em todos os níveis. Apesar da análise aqui realizada ser de corte transversal, é possível deduzir esta questão pelos coeficientes obtidos. Isso mostra que a educação perde um pouco sua importância na renda do trabalhador de um período para o outro, mas ainda assim o nível de escolaridade afeta de forma positiva no salário. De acordo com a Tabela 4, assim como em 2012 a variável escolaridade tem uma maior influência na renda masculina. Nota-se que a renda das mulheres com três anos de estudo cresce percentualmente menos em relação às analfabetas do que a renda das mulheres com dois anos de estudo.

Enquanto no primeiro trimestre de 2012 a experiência interferia de forma mais relevante no salário das mulheres do que dos homens, em 2015 ela se comportou de maneira inversa. Para dois ou mais anos de trabalho houve uma variação de $17,8 \%$ de um período para o outro na renda dos homens, já na renda das mulheres houve uma queda de $14,02 \%$ no mesmo período. Mesmo com essa queda, a experiência continua sendo um coeficiente positivo na variação da renda feminina.

A diferença na variação do salário entre homens e mulheres reduziu no primeiro trimestre de 2015. A renda feminina é aproximadamente 37,4\% menor do que a masculina. Vale ressaltar que mesmo com uma estatística considerável alta, esse número tem se reduzido desde 2012, observa-se que a variação sofreu uma queda de $8 \%$. Em relação à cor ou raça, os amarelos apresentam a maior variação positiva da categoria, ganham cerca de $16,3 \%$ a mais do que os brancos, enquanto os negros, pardos e indígenas têm renda inferior aos brancos.

Para ambos os sexos o setor de atividade tanto para maiores rendimentos quanto para menores manteve-se os mesmos. As atividades mal definidas apresentaram valores menores do que o setor de agropecuária, porém o p-valor é maior que $5 \%$ e os dados não têm significância.

Outro fator a ser observado é o comportamento da variação nos coeficientes da região. As variáveis se mantiveram na mesma ordem, mas pode-se observar que o salário na região Norte caiu, isso porque o Nordeste que é o único com coeficientes menores, conseguiu diminuir essa diferença, e as outras regiões com coeficientes positivos aumentaram ainda mais essa diferença. 
Tabela 5 - Análise da equação minceriana para o primeiro trimestre de 2019

\begin{tabular}{|c|c|c|c|}
\hline Lnsalário & População & Homens & Mulheres \\
\hline Escolaridade & \multicolumn{3}{|c|}{ Coeficientes } \\
\hline \multicolumn{4}{|l|}{ Sem instrucão e menos de 1 ano de } \\
\hline 1 ano de estudo & $0,058^{*}$ & $0,073^{*}$ & $0,028^{\star *}$ \\
\hline 2 anos de estudo & $0,097^{*}$ & $0,119^{*}$ & $0,047^{* *}$ \\
\hline 3 anos de estudo & $0,140^{*}$ & $0,160^{*}$ & $0,108^{*}$ \\
\hline 4 anos de estudo & $0,263^{*}$ & $0,281^{*}$ & $0,241^{*}$ \\
\hline 5 anos de estudo & $0,347^{*}$ & $0,365^{*}$ & $0,332^{*}$ \\
\hline 6 anos de estudo & $0,454^{*}$ & $0,470^{*}$ & $0,447^{\star}$ \\
\hline 7 anos de estudo & $0,520^{*}$ & $0,540^{*}$ & $0,505^{*}$ \\
\hline 8 anos de estudo & $0,529^{*}$ & $0,543^{*}$ & $0,530^{*}$ \\
\hline 9 anos de estudo & $0,596^{*}$ & $0,606^{*}$ & $0,601^{*}$ \\
\hline 10 anos de estudo & $0,591^{*}$ & $0,608^{*}$ & $0,584^{*}$ \\
\hline 11 anos de estudo & $0,613^{*}$ & $0,619^{*}$ & $0,624^{*}$ \\
\hline 12 anos de estudo & $0,813^{*}$ & $0,817^{*}$ & $0,814^{*}$ \\
\hline 13 anos de estudo & $0,893^{*}$ & $0,904^{*}$ & $0,880^{*}$ \\
\hline 14 anos de estudo & $1,024^{*}$ & $1,056^{*}$ & $0,988^{*}$ \\
\hline 15 anos de estudo & $1,033^{*}$ & $1,030^{*}$ & $1,027^{*}$ \\
\hline 16 anos de estudo & $1,517^{*}$ & $1,547^{*}$ & $1,486^{\star}$ \\
\hline \multicolumn{4}{|l|}{ Experiência } \\
\hline \multicolumn{4}{|l|}{ Menos de 1 mês ${ }^{* * *}$} \\
\hline De 1 mês a menos de 1 ano & $0,347^{*}$ & $0,401^{*}$ & $0,263^{*}$ \\
\hline De 1 ano a menos de 2 anos & $0,476^{*}$ & $0,522^{*}$ & $0,406^{*}$ \\
\hline 2 anos ou mais & $0,654^{*}$ & $0,684^{*}$ & $0,615^{\star}$ \\
\hline Idade & $0,007^{*}$ & $0,009^{*}$ & $0,004^{*}$ \\
\hline \multicolumn{4}{|l|}{ Sexo } \\
\hline \multirow{2}{*}{\multicolumn{4}{|c|}{$\begin{array}{l}\text { Homens } \\
\text { Mulheres } \\
\text { " }\end{array}$}} \\
\hline & & & \\
\hline \multicolumn{4}{|l|}{ Raça } \\
\hline \multicolumn{4}{|l|}{ Branca $^{\star \star \star}$} \\
\hline Preta & $-0,086^{*}$ & $-0,086^{*}$ & $-0,088^{*}$ \\
\hline Amarela & $0,061^{*}$ & $0,009^{* *}$ & $0,126^{\star}$ \\
\hline Parda & $-0,109^{*}$ & $-0,105^{\star}$ & $-0,117^{*}$ \\
\hline Indígena & $-0,099^{*}$ & $-0,101^{*}$ & $-0,103^{*}$ \\
\hline \multicolumn{4}{|l|}{ Setor de atividade } \\
\hline \multicolumn{4}{|l|}{ Agricultura, pecuária, produção } \\
\hline Indústria geral & $0,396^{*}$ & $0,471^{*}$ & $0,348^{*}$ \\
\hline Construção & $0,282^{*}$ & $0,264^{*}$ & $0,544^{*}$ \\
\hline Comércio, reparação de veículos & $0,289^{*}$ & $0,281^{*}$ & $0,389^{*}$ \\
\hline Transporte, armazenagem e correio & $0,427^{*}$ & $0,401^{*}$ & $0,630^{*}$ \\
\hline Alojamento e alimentação & $0,219^{*}$ & $0,148^{*}$ & $0,373^{*}$ \\
\hline Informação, comunicação e atividades & $0,470^{*}$ & $0,407^{*}$ & $0,642^{*}$ \\
\hline Administração pública, defesa e & $0,777^{*}$ & $0,749^{*}$ & $0,910^{*}$ \\
\hline Educação, saúde humana e serviços & $0,522^{*}$ & $0,468^{*}$ & $0,647^{\star}$ \\
\hline Outros Serviços & $0,124^{*}$ & $0,118^{*}$ & $0,220^{*}$ \\
\hline Serviços domésticos & $0,131^{*}$ & $0,067^{*}$ & $0,252^{*}$ \\
\hline Atividades mal definidas & $0,269^{*}$ & $0,187^{*}$ & $0,547^{\star}$ \\
\hline \multicolumn{4}{|l|}{ Região } \\
\hline \multicolumn{4}{|l|}{ Norte $^{\star * \star}$} \\
\hline Nordeste & $-0,113^{*}$ & $-0,100^{*}$ & $-0,126^{*}$ \\
\hline Sudeste & $0,335^{\star}$ & $0,336^{*}$ & $0,327^{*}$ \\
\hline Sul & $0,397^{*}$ & $0,413^{*}$ & $0,372^{*}$ \\
\hline Centro-Oeste & $0,394^{*}$ & $0,444^{*}$ & $0,326^{*}$ \\
\hline Constante & $5,264^{*}$ & $5,137^{*}$ & $4,998^{*}$ \\
\hline
\end{tabular}

Fonte: Elaborado pelos autores conforme os dados da PNAD de 2019. ${ }^{*} p$-valor menor que 0,05 (significante). ${ }^{* \star} \mathrm{p}$-valor maior que 0,05 (não significante). ${ }^{* * \star}$ Categorias de referência. 
Com dados do primeiro trimestre de 2019 pode-se notar que a variável escolaridade vem tendo comportamento parecido ao longo dos anos, existe uma queda na importância do estudo para a definição do salário a cada período analisado. Este fator pode ser explicado pelo cenário econômico do período, já que se trata de um ano com menor nível de atividade econômica. Apesar disto, conforme, Ribeiro (2017), diante da diminuição do nível de atividade econômica e da expansão do desemprego, espera-se que a escolaridade desempenhe um papel de influência na inserção e manutenção do indivíduo no mercado de trabalho. Outro fator interessante é que neste ano a variável é crescente em cada ano de estudo a partir do primeiro, diferente dos outros anos.

A importância da experiência vem crescendo, enquanto em 2012 os homens com dois ou mais anos de experiência ganhavam cerca de $44 \%$ a mais do que os indivíduos com menos de um mês de trabalho e as mulheres aproximadamente 45\%. Em 2019 os homens aumentam seu salário em cerca de $68 \%$ e as mulheres $61 \%$. Um aumento aproximado de 54,5\% para a experiência masculina e 35\%para feminina de um período para o outro. Sendo um dos fatores principais na variação do salário.

A diferença no salário das mulheres em relação ao dos homens vem diminuindo gradativamente, de acordo com os dados da tabela em 2019 as mulheres recebem cerca de $35 \%$ menos que os homens com tudo mais constante.

Em relação às raças verifica-se que a conclusão dos dados não se alterou em 2019, ou seja, os amarelos ganham 6,1\% a mais do que os brancos, enquanto os negros, pardos e os indígenas tem renda inferior aos brancos. Quando se analisa essa variável comparando os sexos, observa-se que as mulheres passam a ser mais afetadas do que homens.

Ao averiguar as posições de ocupação, conclui-se que o setor de Administração pública, defesa e seguridade social continua sendo um trabalho mais rentável para ambos os sexos. Em contrapartida, a agropecuária tem sido menos favorável em termos de renda para a população brasileira. Ao comparar as regiões brasileiras, o Norte continua tendo uma queda em sua renda em relação às outras localidades.

Os dados permitem concluir que as mulheres em todos os anos analisados obtiveram um nível de escolaridade maior em relação aos homens, porém a renda 
auferida por elas é menor. Essa argumentação contraria a teoria do capital humano, se comparado ambos os sexos.

Ao analisar separadamente cada sexo pode-se dizer que o aumento da escolaridade tem efeito positivo sobre os rendimentos. Mas, ao comparar os dois sexos, verifica-se que o efeito é maior para os homens do que para as mulheres. Isto fica evidente mesmo com presença das variáveis de controle o que aponta para discriminação no mercado de trabalho em relação às mulheres.

\section{CONSIDERAÇÕES FINAIS}

Na literatura econômica é recorrente a discussão sobre a discriminação das mulheres no mercado de trabalho. Com o passar do tempo verificou-se que a participação feminina no mercado de trabalho tem sofrido mudanças, e isso tem sido positivo para elas, mas ainda está muito aquém do esperado na sociedade. Um dos pontos que colaboraram para essa evolução foi o aumento da escolaridade. Isto, associado à redução da fecundidade possibilitou maior inserção feminina no mercado de trabalho.

O presente estudo teve como objetivo apresentar as diferenças salariais entre mulheres e homens por nível de escolaridade. A hipótese da pesquisa foi que mesmo com um maior grau de escolaridade as mulheres continuam recebendo menores salários. Por meio desse fato demonstra-se que existe discriminação no mercado de trabalho, já que existem grandes diferenças salariais.

Para alcançar a proposta do trabalho, foram utilizados dados da PNAD Contínua dos primeiros trimestres de 2012, 2015 e 2019. Através dos dados foram formuladas estatísticas descritivas e também foi feita uma regressão, por meio da equação minceriana.

Através da análise de dados concluiu-se que as mulheres possuem um nível de escolaridade médio maior que o dos homens, mas a renda média delas é menor em comparação a dos homens. Cabe destacar que a diferença salarial entre os sexos tem se reduzido com o passar dos trimestres analisados. A escolaridade tem aumentado tanto para as mulheres, quanto para os homens, no entanto a dispersão vem diminuindo. 
$\mathrm{Na}$ análise da equação minceriana observou-se que o comportamento das variáveis analisadas nos trimestres escolhidos não mudou. Essa equação permitiu concluir que quanto maior a escolaridade maior é o salário. Apesar disto, em relação aos sexos verificou-se que a escolaridade tem uma interferência maior na renda dos homens, aproximadamente $5,8 \%$. No que diz respeito à experiência quanto maior o tempo de trabalho maior será a variação da renda, isso vale para ambos os sexos. Em relação à idade, a renda dos homens varia aproximadamente $50 \%$ a mais a cada ano de idade se comparado às mulheres.

$\mathrm{Na}$ verificação dos dados relacionados às raças, concluiu-se que essa variável afeta mais os salários dos homens do que as mulheres, isso pode ser explicado pelo fato da maior homogeneidade na renda feminina. Os setores de atividade mais rentáveis para ambos os sexos foram administração pública, defesa e seguridade social. Outra variável que interfere na variação do salário é a região onde mora o indivíduo. Com base na região Norte apenas a região Nordeste possui níveis menores de renda, as outras regiões possuem coeficientes positivos para $O$ rendimento do trabalhador. A variação no salário do homem é maior no CentroOeste e das mulheres na região Sul do país em todo o período analisado na pesquisa.

Os dados mostraram que os rendimentos auferidos pelas mulheres são inferiores aos dos homens, o que sugere discriminação no mercado de trabalho. Pode-se observar que as mulheres, no período atual analisado nesta pesquisa, ainda sofrem discriminação no mercado de trabalho e que ainda há diferença salarial entre os sexos, os dados permitiram essa conclusão. Diante disso, é importante pesquisas sobre o tema de modo a influenciar políticas públicas com intuito de amenizar essa disparidade salarial entre os gêneros. Em pesquisas futuras, pretende-se analisar anualmente os dados e aprofundar algumas variáveis utilizadas neste trabalho, como idade e raça.

\section{REFERÊNCIAS}

ALMEIDA, E. P. de; PEREIRA, R. S. Críticas à teoria do capital humano: uma contribuição à análise de políticas públicas em educação. Revista de Educação

Pública. Cuiabá: UFMT, v. 9, n. 15, 2000.

ANDRADE, Rita de. Teoria do capital humano e a qualidade da educação nos estados brasileiros. 2010. 75f. Trabalho de Conclusão de Curso (Graduação em 
Ciências Econômicas) - Faculdade de Ciências Econômicas, UFGRS, Porto Alegre, 2010.

ARBACHE, J. S. Determinação e diferencial de salários no Brasil. In: FONTES, R.; ARBEX, M. A. (Ed.). Desemprego e mercado de trabalho: ensaios teóricos e empíricos. Viçosa: Editora UFV, p. 125-184, 2000.

BALASSIANO, Moisés; SEABRA, Alexandre Alves de; LEMOS, Ana Heloisa. Escolaridade, salários e empregabilidade: tem razão a teoria do capital humano? Revista de Administração Contemporânea, v. 9, n. 4, p. 31-52, 2005.

BIAGIONI, Daniel. Determinantes da mobilidade por classes sociais: Teoria do Capital Humano e a Teoria da Segmentação do Mercado de Trabalho. In: XV Encontro Nacional de Estudos Populacionais, 2016, Caxambu-MG. Anais..., Belo Horizonte: ABEP, p. 1-19, 2016. Disponível em: < http://www.abep.org.br/publicacoes/index.php/anais/article/view/1501/1466>. Acesso em: 15 de nov. 2019.

BECKER, G. S. Human capital a theoretical and empirical analysis, with special reference to education. Third Edition, University of Chicago, NBER, New York, 1994. Disponível em: <http:// /www.nber.org/books/ beck94-1>. Acesso em: 23 de out. 2019.

BELTRÃO, Kaizô Iwakami. Acesso à educação: diferenciais entre os sexos. Textos para discussão no 879, Rio de Janeiro: IPEA, 2002. Disponível em: < https://www.ipea.gov.br/portal//mages/stories/PDFs/TDs/td_0879.pdf>. Acesso em: 05 de out. 2019.

BRUSCHINI, C.; LOMBARDI, M. R. O trabalho da mulher brasileira nos primeiros anos da década de noventa. In: Encontro Nacional de Estudos Populacionais, ,1996. Caxambu-MG, Anais..., Belo Horizonte: ABEP, p. 483-513, 1996. Disponível em: $<$ http://www.abep.org.br/publicacoes/index.php/anais/article/view/722>. Acesso em: 10 nov. 2019.

BRUSCHINI, Cristina. Gênero e trabalho no Brasil: novas conquistas ou persistência da discriminação. In: ROCHA, M. (Org). Trabalho e Gênero: mudanças, permanências e desafios. Campinas: CEDEPLAR, 384p, p. 13-58, 2000.

BRUSCHINI, Cristina; PUPPIN, Andrea Brandão. Trabalho de mulheres executivas no Brasil no final do século XX. Cadernos de Pesquisa, São Paulo, vol. 34, no 121, p. 105-138, 2004. Disponível em:

< https://www.scielo.br/pdf/cp/v34n121/a06n121.pdf>. Acesso em: 20 de nov. 2019.

IBGE - INSTITUTO BRASILEIRO DE GEOGRAFIA E ESTATÍSTICA. Pesquisa Nacional por Amostras de Domicílios. Rio de Janeiro: IBGE, 2019. Disponível em: $<$ https://www.ibge.gov.br/estatisticas/multidominio/condicoes-de-vida-desigualdadee-pobreza/17270-pnad-continua.html>. Acesso em: 23 de nov. 2019. 
LEME, C.; WAJNMAN, S.. Tendências de coorte nos diferenciais de rendimentos por sexo. In: Henriques, R. (Org.). Desigualdade e pobreza no Brasil. Rio de Janeiro: IPEA, p. 251-270, 2000.

LIMA, R. Mercado de trabalho: o capital humano e a teoria da segmentação.

Pesquisa e Planejamento Econômico, v. 10, n. 1, p. 217-72,1980. Disponível em: < http://repositorio.ipea.gov.br/bitstream/11058/7030/1/PPE_v10_n1_Mercado.pdf>. Acesso em: 25 nov. 2019.

LOUREIRO, Paulo RA. Uma resenha teórica e empírica sobre economia da discriminação. Revista Brasileira de Economia, v. 57, n. 1, p. 125-157, 2003. Disponível em: < scielo.br/pdf/rbe/v57n1/a05v57n1.pdf>. Acesso em: 10 de out. 2019.

MAGALHÃES, Belmira; SILVA, Geice. A mulher no trabalho, na família e na universidade. Revista Eletrônica Arma da Critica, v. 2, n. 2, p. 177-193, 2010. Disponível em: < http://www.armadacritica.ufc.br/phocadownload/12$\% 20$ a\%20mulher\%20no\%20trabalho\%20na\%20familia\%20e\%20na\%20universidade -\%20belmira\%20magalhaes\%20e\%20geice\%20silva.pdf>. Acesso em: 16 nov. 2019.

MINCER, Jacob. Schooling, Experience, and Earnings. National Bureau of Economic Research, Columbia University Press: New York, 1974.

OLIVEIRA, Felipe Resende et al. Salário, educação e experiência: Uma abordagem econométrica. Revista Nexos Econômicos, v. 9, n. 2, p. 7-20, 2015. Disponível em: $<$ https://cienciasmedicasbiologicas.ufba.br/index.php/revnexeco/article/viewFile/1394 4/14047>. Acesso em: 26 nov. 2019.

PROBST, E. R; RAMOS, P. A evolução da mulher no mercado de trabalho. Revista Leonardo Pós. (Instituto Catarinense de Pós-Graduação), p. 35-38, 2003.

RIBEIRO, M. G. Desigualdades de renda: a escolaridade em questão. Educação \& Sociedade. Campinas, v. 38, №. 138, p.169-188, 2017. Disponível em:

< https://www.scielo.br/pdf/es/v38n138/1678-4626-es-ES0101

73302016154254.pdf>. Acesso em: 20 nov. 2019.

SANTOS, Renato Vale; RIBEIRO, Eduardo Pontual. Diferenciais de rendimentos entre homens e mulheres no Brasil revisitado: explorando o "teto de vidro". Rio de Janeiro, UFRJ (Texto para Discussão), p. 1-34, 2006. Disponível em: <http://www.sebh.ecn.br/seminario_4/arquivo5.pdf>. Acesso em: 15 out. 2019.

SILVA, Vitor Hugo Miro Couto; FRANÇA, João Mário Santos de; PINHO NETO, Valdemar Rodrigues de. Capital humano e desigualdade salarial no Brasil: uma análise de decomposição para o período 1995-2014. Estudos Econômicos (São Paulo), v. 46, n. 3, p. 579-608, 2016. mercado de trabalho brasileiro: 1995, 2001, 2006 e 2012. 2014. 36f. Trabalho de 
Conclusão de Curso (Graduação em Ciências Econômicas) - Setor de Ciências Sociais Aplicadas, UFP, Curitiba, 2014.

SCHULTZ, T. W. O valor econômico da educação. Rio de Janeiro: Zahar Editores, 1964.

SOUZA, Solange de Cassia Inforzato et al. Diferenças salariais por gênero e cor e o impacto da discriminação econômica. Revista Brasileira de Estudos Regionais e Urbanos, v. 9, n. 1, p. 32-49, 2015. Disponível em:

<https://revistaaber.org.br/rberu/article/view/129>. Acesso em: 20 out. 2019.

Recebido em maio de 2020

Aceito em julho de 2020 